\title{
Catheter Ablation of Incisional Atrial Tachycardia
}

\author{
Roman Tatarskiy, Svetlana Garkina, Dmitriy Lebedev \\ Federal Almazov North-West Medical Research Centre, Saint Petersburg, Russia.
}

\begin{abstract}
Tachycardias after atrial incisions represent frequent and serious problem. The majority of them are based on a re-entry electrical activation around a combination of anatomic and surgically created obstacles. Considering significant progress of cardiovascular surgery during the last decade along with potential large amount of open-heart procedures in the near future the number of incisional tachycardias has a tendency to increase. The aim of this work was to quantify the magnitude of the problem, characterize the tachycardias after different surgical operations and to analyze possible interventional treatment strategies. Nowadays evolution of mapping and ablation technologies may contribute to radically treatment of this type of arrhythmias while there are still a lot of issues that should be solved to improve the results of interventional treatment of incisional tachycardias.
\end{abstract}

\section{Introduction}

Atrial incisional arrhythmias represent a common complication of cardiovascular surgery. The term incisional atrial tachycardia (intraatrial re-entry tachycardia) is used when the re-entry zone is localized around postoperative scar. Considering significant progress of cardiovascular surgery during the last decade along with potential large amount of open-heart procedures in the near future the number of incisional tachycardias has a tendency to increase. Beyond that, heart surgery associated with manipulation inside the atria, such as correction of congenital malformations, valve interventions or "maze" procedure may appear the common cause of atrial tachycardia. Such atrial arrhythmias are caused by myocardial damage and mostly demonstrate macro-re entry mechanism. ${ }^{1-4}$ We can't also underestimate the role of atrial fibrosis, pericardial inflammation and high blood pressure in cardiac chambers. These factors cause a dispersion of myocardial refractory and sinus node dysfunction and contribute to atrial conduction delay that may predispose to the formation of re-entry. Cardiopulmonary bypass surgery, metabolic and electrolyte disturbances, increased adrenergic tone and use of inotropic agents contribute to high risk of arrhythmias during perioperative period. Studies demonstrated that small body weight, young age, prolonged cardiopulmonary bypass time, the complexity

Key Words:

Catheter Ablation, Incisional Tachycardia, Electroanatomical Mapping, Substrate Mapping.

Disclosures:

None.

Corresponding Author:

Roman Tatarskiy,

Federal Almazov North-West Medical Research Centre,

Saint Petersburg, 197341,

Russia. of the surgery and the residual defect were the risk factors of early postoperative arrhythmias. ${ }^{5,6}$ According to experts opinion, incisional atrial tachycardia occurs in $10-30 \%$ of patients after magistral vessels transposition and in $20-37 \%$ of patients undergoing the Fontan operation..$^{1-4}$ According to data of M.Gelatt et al., up to $14 \%$ among 478 patients after Mustard procedure presented incisional atrial arrhythmias while $1 \%$ demonstrated ectopic tachycardias. Overall prevalence of incisional tachycardias during 20 years of follow up was 24\%.7 Atrial incisions performed during surgical correction of Fallot's tetralogy also predispose to further incisional tachycardia where $10 \%$ of patients experience atrial arrhythmias, $11 \%$ demonstrate sustained ventricular tachycardia and $8 \%$ of patients die suddenly. ${ }^{8-10}$ The loss of coordinated atrial activity and the increase of rate frequency may be accompanied by severe disorders of systemic hemodynamics. It was reported that atrial arrhythmias leading to deterioration of ventricular function were associated with increased overall risk of death including sudden cardiac death. ${ }^{11,12} \mathrm{It}$ was shown that right atrium surgery during septal defects closure or correction of other congenital heart anomalies represent another major cause of atrial re-entry arrhythmias. ${ }^{13-20}$ Such tachycardias tend to have various localization due to individual anatomical features and surgical technique as well as the severity of atrial fibrosis. Incisional tachycardia can occur both during early and late postoperative period. Early postoperative arrhythmias often require correction of electrolyte balance along with pharmacological and non-pharmacological interventions. Late postoperative arrhythmias are associated with numerous risk factors, including direct injury of cardiac conduction system, the surgical "scars" that contribute to conduction abnormalities; and a combination of hemodynamic, anatomical and electrical disturbances in patients with structural heart disease. Modified Fontan procedure and prior early atrial tachycardia are also considered to be independent predictors of late 
incisional arrhythmias. ${ }^{21}$

Patients with sinus node dysfunction also demonstrate high prevalence of atrial tachycardias. Both early and late postoperative arrhythmias represent an important risk factor for morbidity and mortality after cardiac surgery. Typical (isthmus-dependent) atrial flutter is the most common arrhythmia in patients undergoing heart surgery, often combined with incisional atrial tachycardias, forming several re-entry zones or complex dual-loop (figure-eight) re-entry circuit. $^{22,23}$ Right atrial re-entry mechanisms are more frequent compared to left atrium. ${ }^{1,24,25}$ According to W.Anne et al., the majority of patients selected for atrial incisional tachycardia ablation presented right-side arrhythmias including typical atrial flutter (62\%) while only $49 \%$ of overall rate were associated with atriotomy. However, left-sided arrhythmias were reported to be more common among the patients with acquired valvular disease. ${ }^{26}$ Atrial ectopic tachycardia may also complicate the postoperative period after heart surgery. Unlike atrial re-entry tachycardia, it has different cycle lengh, usually starts and ends slowly, and does not respond to stimulation in «overdrive pacing» mode. ${ }^{23}$ The prevalence of postoperative ectopic tachycardia varies according to different authors from 1 to $50 \%{ }^{27-30}$ Most often, it occurs after correction of Fallot's tetralogy or interventions near atrioventricular node. ${ }^{31}$ Thus, data from A. DodgeKhatami et al. showed $11 \%$ rate of atrioventricular ectopy after correction of congenital heart defects whereas overall mortality was reported about $3 \% .{ }^{29-31}$ The exact cause of ectopic nodal tachycardia is unknown, but it can be assumed that the increase of His automatism is associated with its surgical damage. Meanwhile, direct correlation between the ectopic nodal tachycardia and young age along with lack of magnesium in the postoperative period was revealed. ${ }^{32}$

Due to variety of potential arrhythmias after cardiac surgery it is sometimes difficult to adequately differentiate them from each other. In most cases, the absence of distinct atrial activity on the ECG leads to confusion and misdiagnosis of atrial fibrillation. Large P-waves on the surface electrocardiogram are often mistaken for typical atrial flutter. Unlike the classical type flutter with saw-toothed P-waves and heart rate about 300 beats per minute, atrial incisional tachycardias often demonstrate slow rhythm with different $\mathrm{P}$-waves pattern. These deviations correlate with different excitation wavefront spreading and scar location in the atria. Sometimes $\mathrm{P}$-waves during incisional tachycardia may be similar to typical pattern of isthmus-dependent atrial flutter. ${ }^{1}$ In some cases, it is difficult to identify the $\mathrm{P}$-wave on the surface ECG, which is probably due to small scar size and short circle of re-entry. Thus, diagnostic intracardiac electrophysiological study is required. However, it is generally possible to identify incisional atrial tachycardia on surface ECG. It is important that re-entry zone is associated with scars as result of surgery. Functionally, they represent lines of conduction block. It should be noted that functional blockade doesn't always correlate with anatomical substrate as confirmed by autopsy. Area of functional block is characterized as full electric "silence" or fragmented and low voltage electrograms. The number of possible re-entry circles is clearly associated with the number of isolated "channels". It was shown by H.Nakagawa et al., that patients after Fontan procedure had much more myocardial "channels" in contrast to those after intratrial septal occlusion or Fallot's tetralogy interventions and this fact predisposes to multiple atrial re-entry tachicardias. ${ }^{2}$

In other single center study M.N.S. De Groot et al. analyzed the voltage of intracardiac electrograms in patients with congenital heart anomalies compared to healthy individuals. It was found that the amplitude of atrial potential in case of congenital structural diseases was significantly lower than in normal hearts. Localization of lowamplitude electrograms correlated with re-entry zone. ${ }^{33} \mathrm{~B}$. Love et al. reported association between some electrical parameters (double potentials, electrical "silence" region) and central re-entry circuit in patients with congenital heart anomalies during sinus rhythm, atrial pacing and during tachycardia. ${ }^{33}$ Therefore, a correlation between electrical activity and localization of tachycardia is demonstrated. However, data about electrical and histopathological correlation in patients with atrial incisional tachycardias are lacking, due to technical difficulties and short follow up period.

During surgery interventions, atriotomy, cannulation or other manipulations may lead to postoperative scar formation, usually in lateral wall of right, while re-entry wavefront circulates around the incision. ${ }^{2}$ Linear ablation from the bottom edge of scar to inferior vena cava or tricuspid valve, or from the top edge of scar to superior vena cava provides re-entry circuit interruption. ${ }^{34-35}$ Sometimes scar heterogeneity leads to "channel" formation between high-density areas resulting into slow and fragmented electrical activity that is necessary for re-entry mechanism. When there are a lot of "channels", various forms of tachycardias may be induced. Thus, termination of "channels" activity leads to the elimination of tachycardia. ${ }^{2}$ Atrial electroanatomical mapping often identifies areas with low amplitude of atrial potentials, including distant sites far from surgical lesions. This fact may indicate large zone of myocardial damage or atrial wall infarction while definite etiology of "atrial myopathy" remains unclear. It possibly can be explained by the interruption of atrial blood supply and the lack of protection during cardioplegia. The use of antiarrhythmic drugs and atrial pacing didn't demonstrate effectiveness in the incisional tachycardia treatment. ${ }^{36,37}$ Furthermore, drug therapy is limited by potential organotoxic complications and risk of pro-arrhythmic activity. Antitachycardia pacing is usually used for re-entry arrhythmia termination but in case of atrial incisional tachycardias may often induce atrial fibrillation with subsequent deterioration of hemodynamics. ${ }^{37}$ Only intracardiac electrophysiological study and radiofrequency ablation may potentially modify the vulnerable section of re-entry circuit and prevent arrhythmia recurrence. Several studies on young patients after surgical correction of congenital heart anomalies demonstrated average efficacy of radiofrequency ablation from 12 to $50 \%$ during more than 2 years of follow-up.,14-16

The lack of randomized clinical trials is the main limitation when the effectiveness of incisional tachycardia treatment is estimated. The termination of arrhythmia during ablation doesn't always represent adequate clinical point of radical treatment. The problem of frequent recurrence after successful ablation of tachycardia requires further evaluation and formation of additional predictors of effectiveness. ${ }^{38}$

Non-fluoroscopic ablation without atrial activation mapping may provide tachycardia termination but doesn't allow to estimate conduction block inside the vulnerable re-entry zone. According to the North American Pediatric Registry of radiofrequency ablation, the effectiveness of procedure was only $55 \%$ in the mid-1990s while other centers reported up to $78 \%$ efficacy. ${ }^{34,35,39-42}$ During 2-year follow-up after successful tachycardia ablation $50 \%$ rate of arrhythmia recurrence was demonstrated. ${ }^{38}$

As reported by W.Anne et al., using of Halo catheter with the possibility of registering more than 20 endocardial electrograms 
allowed to identify re-entry zones and improve the efficiency of tachycardia ablation up to $94 \%$. But at the same time the rate of post-operative recurrence was $29 \%$ that could be explained by new re-entry zones formation. There was direct correlation between the number of re-entry curcuits and risk of arrhythmia recurrence after first ablation procedure. Long-term success rate depends on number of different types of tachycardia and corresponds with other literature data. ${ }^{18,35}$ More frequent recurrence of atrial incisional arrhythmias (33-53\%) in early studies was associated with inability to identify all potentially vulnerable areas of re-entry, especially in patients with multiple "scars" in the myocardium. , $35,37,42$

Radiofrequency ablation of incisional atrial re-entry tachycardia is complicated by several factors and requires:

1. Identification of vulnerable tachycardia zones.

2. Formation of effective transmural lesions.

3. Verification of bidirectional conduction block.

4. Confirmation the lack of recurrent episodes during long-term follow-up.

A detailed understanding of the incisional tachycardias mechanisms, and therefore, successful treatment is obtained using nonfluoroscopic mapping systems with real-time analysis and graphic 3D visualization of myocardial activation. Contemporary navigation systems provide high-accuracy data (estimated error no more than $1 \mathrm{~mm}$ ) on three-dimensional geometrical reconstruction of the heart chambers and electroanatomical mapping. It is safe and informative technique for atrial activation visualization in patients with complex incisional atrial arrhythmias, which contributes to diagnosis of both focal and incisional atrial reentry tachycardia. Analysis of activation map may help to identify critical area of slow conduction, contributing to successful catheter ablation. Visualization of surgical scars and anatomical structural barriers within the threedimensional electroanatomical map simplifies the process of linear lesions creation. ${ }^{43}$ Electroanatomical mapping allows to differentiate ectopic and re-entry tachycardia. This method is wide practically used and represents a systematic approach to the incisional tachycardia treatment Long-term outcome of ablative therapy of post-operative atrial tachyarrhythmias in patients with tetralogy of Fallot: a European multi-centre study. ${ }^{4-46}$

Electroanatomical mapping during sustained tachycardia helps to identify zones scar in the myocardium, "channels" of slow conduction or the phenomenon of "snake biting its own tail", which is characterized as persistence of the earliest and most recent activation in the tachycardia circuit. After failing to induce the tachycardia or in cases of unstable tachycardia or various cycle length, mapping can be performed during atrial pacing, to detect slow conduction "channels" between scars and normal anatomical structures. Radiofrequency energy should be delivered at specific sites demonstrating "abnormal" voltage, isthmus of a circuit, or slow conduction zones. ${ }^{47}$ The main principle of procedure is modifying the arrhythmogenic substrate connecting scar/abnormal myocardium to natural anatomical barriers (upper and lower vena cava, tricuspid and mitral rings) or another scar with tachycardia elimination. Main criteria of transmural necrosis during ablation include low amplitude (up to $80 \%$ of baseline) or fragmentation of unipolar atrial potential, registered with map catheter. ${ }^{2}$ Potential complications of ablation procedure include phrenoplegia, caused by phrenic nerve damage, and thromboembolic events after conversion to sinus rhythm. More rarely occur hemopericardium, cardiac tamponade and atrio- esophageal fistulas.

Comparative study at Boston Children's Hospital demonstrated significant increase of incisional tachycardia ablation efficacy using electroanatomical mapping. ${ }^{48}$ If entrainment pacing was not performed, it didn't reduce effectiveness of the procedure. ${ }^{2,37}$ Under $3 \mathrm{D}$ mapping guidance the rate of successful ablation is over $90 \%{ }^{2,49}$

\section{Conclusions}

Therefore, improved surgical techniques during last decades contributes to better prognosis in patients with congenital heart anomalies and acquired valvular disease while the risk of incisional atrial tachycardias is progressively increasing. On the other hand, growing number of patients with repeated surgical procedures leads to multiple scars formation in the atria and consistently escalates risk of arrhythmias. High rate of postoperative arrhythmias and their complications stimulates the development of new interventional techniques. Understanding the electrophysiological mechanisms and evolution of mapping and ablation technologies will contribute to radically treatment of this type of arrhythmias. However, there are still a lot of issues that should be solved to improve the results of surgical treatment of incisional arrhythmias.

\section{References}

1. Page Richard L, JoglarJosé A, CaldwellMary A, CalkinsHugh, ContiJamie B, DealBarbara J, Estes IiiN A Mark, FieldMichael E, GoldbergerZachary D, HammillStephen C, IndikJulia H, LindsayBruce D, OlshanskyBrian, RussoAndrea M, ShenWin-Kuang, TracyCynthia M, Al-KhatibSana M. 2015 ACC/AHA/HRS guideline for the management of adult patients with supraventricular tachycardia: Executive summary: A Report of the American College of Cardiology/American Heart Association Task Force on Clinical Practice Guidelines and the Heart Rhythm Society. Heart Rhythm. 2016;13 (4):e92-135.

2. Nakagawa H, ShahN, MatsudairaK, OverholtE, ChandrasekaranK, BeckmanK J, SpectorP, CalameJ D, RaoA, HasdemirC, OtomoK, WangZ, LazzaraR, JackmanW M. Characterization of reentrant circuit in macroreentrant right atrial tachycardia after surgical repair of congenital heart disease: isolated channels between scars allow "focal" ablation. Circulation. 2001;103 (5):699-709.

3. Shah D, JaisP, TakahashiA, HociniM, PengJ T, ClementyJ, HaïssaguerreM. Dualloop intra-atrial reentry in humans. Circulation. 2000;101 (6):631-9.

4. Triedman J K, AlexanderM E, BerulC I, BevilacquaL M, WalshE P. Electroanatomic mapping of entrained and exit zones in patients with repaired congenital heart disease and intra-atrial reentrant tachycardia. Circulation. 2001;103 (16):2060-5.

5. Valsangiacomo Emanuela, SchmidEdith R, SchüpbachRolf W, SchmidlinDaniel, MolinariLuciano, WaldvogelKatharina, BauersfeldUrs. Early postoperative arrhythmias after cardiac operation in children. Ann. Thorac. Surg. 2002;74 (3):792-6.

6. Pfammatter Jean-Pierre, WagnerBendicht, BerdatPascal, BachmannDenis C G, PavlovicMladen, PfenningerJürg, CarrelThierry. Procedural factors associated with early postoperative arrhythmias after repair of congenital heart defects. J. Thorac. Cardiovasc. Surg. 2002;123 (2):258-62.

7. Gelatt M, HamiltonR M, McCrindleB W, ConnellyM, DavisA, HarrisL, GowR M, WilliamsW G, TruslerG A, FreedomR M. Arrhythmia and mortality after the Mustard procedure: a 30-year single-center experience. J. Am. Coll. Cardiol. 1997;29 (1):194-201.

8. Gatzoulis M A, BalajiS, WebberS A, SiuS C, HokansonJ S, PoileC, RosenthalM, NakazawaM, MollerJ H, GilletteP C, WebbG D, RedingtonA N. Risk factors for arrhythmia and sudden cardiac death late after repair of tetralogy of Fallot: a multicentre study. Lancet. 2000;356 (9234):975-81. 
9. Li W, SomervilleJ. Atrial flutter in grown-up congenital heart $(\mathrm{GUCH})$ patients. Clinical characteristics of affected population. Int. J. Cardiol. 2000;75 (2-3):12937.

10. Li W, SomervilleJ, GibsonD G, HeneinM Y. Disturbed atrioventricular electromechanical function long after Mustard operation for transposition of great arteries: a potential contributing factor to atrial flutter. J Am Soc Echocardiogr. 2001;14 (11):1088-93.

11. de Groot Natasja M S, LukacPeter, SchalijMartin J, MakowskiKarol, SziliTorokTamas, JordaensLuc, NielsenJens Cosedis, JensenHenrik Kjaerulf, GerdesJens Christian, DelacretazEtienne. Long-term outcome of ablative therapy of post-operative atrial tachyarrhythmias in patients with tetralogy of Fallot: a European multi-centre study. Europace. 2012;14 (4):522-7.

12. Papagiannis John, MaounisThemistoklis, LaskariCleo, TheodorakisGeorge N, RammosSpyridon. Ablation of atrial tachycardias with radiofrequency current after surgical repair of complex congenital heart defects. Hellenic J Cardiol. 2007;48 (5):268-77.

13. Scaglione Marco, CaponiDomenico, EbrilleElisa, Di DonnaPaolo, Di ClementeFrancesca, BattagliaAlberto, RaimondoCristina, AppendinoManuela, GaitaFiorenzo. Very long-term results of electroanatomic-guided radiofrequency ablation of atrial arrhythmias in patients with surgically corrected atrial septal defect. Europace. 2014;16 (12):1800-7.

14. Akar J G, KokL C, HainesD E, DiMarcoJ P, MounseyJ P. Coexistence of type $\mathrm{I}$ atrial flutter and intra-atrial re-entrant tachycardia in patients with surgically corrected congenital heart disease. J. Am. Coll. Cardiol. 2001;38 (2):377-84.

15. Chan D P, Van HareG F, MackallJ A, CarlsonM D, WaldoA L. Importance of atrial flutter isthmus in postoperative intra-atrial reentrant tachycardia. Circulation. 2000;102 (11):1283-9.

16. Delacretaz E, GanzL I, SoejimaK, FriedmanP L, WalshE P, TriedmanJ K, SlossL J, LandzbergM J, StevensonW G. Multi atrial maco-re-entry circuits in adults with repaired congenital heart disease: entrainment mapping combined with threedimensional electroanatomic mapping. J. Am. Coll. Cardiol. 2001;37 (6):1665-76.

17. Duru F, HindricksG, KottkampH. Atypical left atrial flutter after intraoperative radiofrequency ablation of chronic atrial fibrillation: successful ablation using three-dimensional electroanatomic mapping. J. Cardiovasc. Electrophysiol. 2001;12 (5):602-5.

18. Hebe J, HansenP, OuyangF, VolkmerM, KuckK H. Radiofrequency catheter ablation of tachycardia in patients with congenital heart disease. Pediatr Cardiol. 2000;21 (6):557-75.

19. Kall J G, RubensteinD S, KoppD E, BurkeM C, VerdinoR J, LinA C, JohnsonC T, CookeP A, WangZ G, FumoM, WilberD J. Atypical atrial flutter originating in the right atrial free wall. Circulation. 2000;101 (3):270-9.

20. Molenschot M, RamannaH, HoorntjeT, WittkampfF, HauerR, DerksenR, SreeramN. Catheter ablation of incisional atrial tachycardia using a novel mapping system: LocaLisa. Pacing Clin Electrophysiol. 2001;24 (11):1616-22.

21. Cecchin F, JohnsrudeC L, PerryJ C, FriedmanR A. Effect of age and surgical technique on symptomatic arrhythmias after the Fontan procedure. Am.J. Cardiol. 1995;76 (5):386-91.

22. Liuba Ioan, JönssonAnders, WalfridssonHakan. Figure- 8 tachycardia confined to the anterior wall of the left atrium. Indian Pacing Electrophysiol J. 2004;4 (3):146-51.

23. Lan Yueh-Tze, LeeJoselyn C R, WetzelGlenn. Postoperative arrhythmia. Curr. Opin. Cardiol. 2003;18 (2):73-8.

24. Jaïs P, ShahD C, HaïssaguerreM, HociniM, PengJ T, TakahashiA, GarrigueS, Le MétayerP, ClémentyJ. Mapping and ablation of left atrial flutters. Circulation. 2000;101 (25):2928-34.

25. Tai C T, LinY K, ChenS A. Atypical atrial flutter involving the isthmus between the right pulmonary veins and fossa ovalis. Pacing Clin Electrophysiol. 2001;24 (3):384-7.
26. Anné W, van RensburgH, AdamsJ, EctorH, Van de WerfF, HeidbüchelH. Ablation of post-surgical intra-atrial reentrant tachycardia. Predilection target sites and mapping approach. Eur. Heart J. 2002;23 (20):1609-16.

27. Entenmann Andreas, MichelMiriam, EgenderFriedemann, HesslingVera, KramerHans-Heiner. Impact of Different Diagnostic Criteria on the Reported Prevalence of Junctional Ectopic Tachycardia After Pediatric Cardiac Surgery. Pediatr Crit Care Med. 2016;17 (9):845-51.

28. Talwar Sachin, PatelKartik, JunejaRajnish, ChoudharyShiv Kumar, AiranBalram. Early postoperative arrhythmias after pediatric cardiac surgery. Asian Cardiovasc Thorac Ann. 2015;23 (7):795-801.

29. Dodge-Khatami A, MillerO I, AndersonR H, Gil-JaurenaJ M, GoldmanA P, de LevalM R. Impact of junctional ectopic tachycardia on postoperative morbidity following repair of congenital heart defects. Eur J Cardiothorac Surg. 2002;21 (2):255-9.

30. Perry J C, FenrichA L, HulseJ E, TriedmanJ K, FriedmanR A, LambertiJ J. Pediatric use of intravenous amiodarone: efficacy and safety in critically ill patients from a multicenter protocol. J. Am. Coll. Cardiol. 1996;27 (5):1246-50.

31. Dodge-Khatami Ali, MillerOwen I, AndersonRobert H, GoldmanAllan P, GilJaurenaJuan Miguel, ElliottMartin J, TsangVictor T, De LevalMarc R. Surgical substrates of postoperative junctional ectopic tachycardia in congenital heart defects. J. Thorac. Cardiovasc. Surg. 2002;123 (4):624-30.

32. Dorman B H, SadeR M, BurnetteJ S, WilesH B, PinoskyM L, ReevesS T, BondB $\mathrm{R}$, SpinaleF G. Magnesium supplementation in the prevention of arrhythmias in pediatric patients undergoing surgery for congenital heart defects. Am. Heart J. 2000;139 (3):522-8.

33. De Groot NMS, BlomNA, van ErverL. 3-D scar tissue mapping to facilitate radiofrequency catheter ablation of post-operative atrial reentrant tachycardia. Pacing. Clin Electrophysiol. 2000;23 (2):578.

34. Love B A, CollinsK K, WalshE P, TriedmanJ K. Electroanatomic characterization of conduction barriers in sinus/atrially paced rhythm and association with intraatrial reentrant tachycardia circuits following congenital heart disease surgery. J. Cardiovasc. Electrophysiol. 2001;12 (1):17-25.

35. Kalman J M, VanHareG F, OlginJ E, SaxonL A, StarkS I, LeshM D. Ablation of 'incisional' reentrant atrial tachycardia complicating surgery for congenital heart disease. Use of entrainment to define a critical isthmus of conduction. Circulation. 1996;93 (3):502-12.

36. Garson A, Bink-BoelkensM, HessleinP S, HordofA J, KeaneJ F, NechesW H, PorterC J. Atrial flutter in the young: a collaborative study of 380 cases. J. Am. Coll. Cardiol. 1985;6 (4):871-8.

37. Ouyang Feifan, ErnstSabine, VogtmannThomas, GoyaMasahiko, VolkmerMarius, SchaumannAnselm, BänschDietmar, AntzMatthias, KuckKarl-Heinz. Characterization of reentrant circuits in left atrial macroreentrant tachycardia: critical isthmus block can prevent atrial tachycardia recurrence. Circulation. 2002;105 (16):1934-42.

38. Triedman J K, BergauD M, SaulJ P, EpsteinM R, WalshE P. Efficacy of radiofrequency ablation for control of intraatrial reentrant tachycardia in patients with congenital heart disease. J. Am. Coll. Cardiol. 1997;30 (4):1032-8.

39. Kanter R J, PapagiannisJ, CarboniM P, UngerleiderR M, SandersW E, WhartonJ M. Radiofrequency catheter ablation of supraventricular tachycardia substrates after mustard and senning operations for $\mathrm{d}$-transposition of the great arteries. J. Am. Coll. Cardiol. 2000;35 (2):428-41.

40. PerryJC,IversonP,KuglerJD. Radiofrequency catheter ablation of tachyarrhythmias in young patients with structurally abnormal hearts. Pacing Clin. Electrophysiol. 1996;19 (2):579.

41. Van Hare G F, LeshM D, RossB A, PerryJ C, DorostkarP C. Mapping and radiofrequency ablation of intraatrial reentrant tachycardia after the Senning or Mustard procedure for transposition ofthe great arteries. Am. J. Cardiol. 1996;77 (11):985-91. 
42. Baker B M, LindsayB D, BrombergB I, FrazierD W, CainM E, SmithJ M. Catheter ablation of clinical intraatrial reentrant tachycardias resulting from previous atrial surgery: localizing and transecting the critical isthmus. J. Am. Coll. Cardiol. 1996;28 (2):411-7.

43. Zhou Gong-bu, HuJi-qiang, GuoXiao-gang, LiuXu, YangJian-du, SunQi, MaJian, OuyangFei-fan, ZhangShu. Very long-term outcome of catheter ablation of post-incisional atrial tachycardia: Role of incisional and non-incisional scar. Int. J. Cardiol. 2016;205 ():72-80.

44. de Groot Natasja M S, LukacPeter, SchalijMartin J, MakowskiKarol, SziliTorokTamas, JordaensLuc, NielsenJens Cosedis, JensenHenrik Kjaerulf, GerdesJens Christian, DelacretazEtienne. Long-term outcome of ablative therapy of post-operative atrial tachyarrhythmias in patients with tetralogy of Fallot: a European multi-centre study. Europace. 2012;14 (4):522-7.

45. Hebe J, AntzM, OuyangF. Mapping and ablation of incisional atrial reentry tachycardias in patients after surgery for congenital heart disease using a 3D-lectroanatomical mapping system. Circulation. 1998;98:616.

46. Dorostkar P C, ChengJ, ScheinmanM M. Electroanatomical mapping and ablation of the substrate supporting intraatrial reentrant tachycardia after palliation for complex congenital heart disease. Pacing Clin Electrophysiol. 1998;21 (9):1810-9.

47. Pap Róbert, MakaiAttila, SághyLászló. Post-incisional right atrial tachycardia eliminated by a single radiofrequency lesion. J Interv Card Electrophysiol. 2007;19 (2):73-6.

48. Collins K K, LoveB A, WalshE P, SaulJ P, EpsteinM R, TriedmanJ K. Location of acutely successful radiofrequency catheter ablation of intraatrial reentrant tachycardia in patients with congenital heart disease. Am. J. Cardiol. 2000;86 (9):969-74.

49. Morady Fred. Catheter ablation of supraventricular arrhythmias: state of the art. Pacing Clin Electrophysiol. 2004;27 (1):125-42. 